\title{
Peripartum Cardiomyopathy: A Cause of Heart Failure in Young Women
}

\author{
Frederick B. Peng, BS and Ryan J. Hidalgo, MD
}

\section{INTRODUCTION}

Peripartum cardiomyopathy (PPCM), also known as postpartum cardiomyopathy, is an infrequent cause of systolic heart failure that can affect women in the last month of pregnancy and up to five months after delivery. While PPCM is a type of dilated cardiomyopathy, the etiology of this condition remains largely unknown. ${ }^{1}$ Given the infrequency with which PPCM is encountered and the potential for this condition to be fatal, proper diagnosis and management is critical for reducing maternal and fetal complications, as well as assessing the safety of future pregnancies.

\section{CASE PRESENTATION}

A 33-year-old gravida 3, para 1, abortus 1 (G3P1011) at 39 weeks and 5 days of gestation presented to labor and delivery with a complaint of new-onset headache and vaginal bleeding that began earlier that morning. Her medical and social histories were significant for chronic hypertension and a one-half pack per day smoking history. Despite persistently elevated blood pressure greater than $140 / 90 \mathrm{mmHg}$, the remainder of an evaluation for preeclampsia was negative. Clinical assessment of her vaginal bleeding was suspicious for abruptio placentae, ultimately leading to the decision to proceed with induction of labor. Subsequent arrest of dilatation and concerns for worsening abruption led to cesarean delivery of a live male. After an uncomplicated recovery, the patient was discharged home on the second postoperative day with a narcotic analgesic and a beta blocker, which was started during her pregnancy and continued as treatment for her chronic hypertension. On the sixth postoperative day, she presented to the emergency department with severe shortness of breath. Physical examination was remarkable for tachycardia, bilateral basilar crackles on chest auscultation, and $1+$ edema in her lower extremities. Chest CTA demonstrated no evidence of pulmonary embolism and cardiac MRI showed no evidence of myocardial scarring, fibrosis, or pericarditis. Her NT-proBNP $(22,555 \mathrm{pg} / \mathrm{ml})$ and cardiac troponin I levels $(0.172 \mathrm{ng} / \mathrm{ml}$ ) were significantly elevated, and she was diagnosed clinically with acute heart failure. Right heart catheterization and tissue biopsy were considered, but not performed. An echocardiogram revealed an estimated left ventricular ejection fraction (LVEF) of 25 to 30\%, consistent with the diagnosis of PPCM.

\section{OUTCOME AND FOLLOW-UP}

The patient was initially treated with diuretics, vasodilators, beta-blockers, and bronchodilators. Despite these interventions, her condition worsened, and she was transferred to the Intensive Care Unit (ICU) for respiratory distress, ultimately requiring non-invasive respiratory assistance with bilevel positive airway pressure (BiPAP). Following lack of clinical improvement over 24 hours and a worsening LVEF of $<20 \%$, she was transferred to our institution and admitted to the cardiac ICU. An ACE-inhibitor and a prophylactic dose of heparin (5000 units subcutaneous injections every 8 hours) were added to her medical regimen due to her risk factors for venous thromboembolism (low ejection fraction and recent pregnancy). She was also treated with aggressive diuresis. After a few days, her dyspnea, lower extremity edema, and pulmonary congestion improved. Once hemodynamically stable, she was transferred to a general medical/cardiology floor and was subsequently discharged with a wearable defibrillator, angiotensin receptor blocker, anticoagulant, diuretics, and instructions to follow-up with outpatient cardiology. The goal was to start her on Entresto (sacubitril/valsartan), which works well in patients with heart failure with reduced ejection fraction. However, the use of Entresto and an ACE-inhibitor is contraindicated, and a washout period of 36 hours is required between administration of the two medications. ${ }^{2}$ The cardiology team also planned for at least three months of prophylactic anticoagulation. The patient was discharged home with warfarin $2.5 \mathrm{mg}$ PO every other day, alternating with $5.0 \mathrm{mg}$ PO on the off-days until she became therapeutic.

\section{DISCUSSION}

PPCM is a serious complication of pregnancy with an incidence that varies geographically, possibly due to socioeconomic and genetic factors. For example, the highest rates of PPCM are reported in Haiti (33 per 10,000 live births), whereas the incidence in the United States is estimated to be 4.84 per 10,000 live births. While the etiology of the condition is unknown, multiple hypotheses have been proposed including a final common pathway that increases oxidative stress and cleaves prolactin into a $16 \mathrm{kDA}$ fragment that results in endothelial damage and myocardial dysfunction. An inflammatory cascade of cytokines (tumor necrosis factor-alpha and interleukin-6) is signaled, which further 
precipitates the condition. Specifically, altered prolactin processing results in a pro-apoptotic, anti-angiogenic, pro-inflammatory, and cardiotoxic 16kDA isoform. ${ }^{3}$ Other small studies have demonstrated an increased prevalence of PPCM with myocarditis and found the presence of parvovirus B19, human herpes virus-6, and human cytomegalovirus (CMV) in myocardial biopsy samples of PPCM patients. ${ }^{4}$ Known risk factors of PPCM include age greater than 30 years, multiple gestations, non-Hispanic African American race, maternal cocaine abuse, and history of preeclampsia, eclampsia, HELLP syndrome, and postpartum hypertension. ${ }^{1.5}$

The signs and symptoms associated with PPCM are similar to that of third-trimester pregnancy, so diagnosis is often delayed. Patients often present with symptoms secondary to volume overload - including dyspnea, cough, orthopnea, paroxysmal nocturnal dyspnea, and pedal edema - which overlap with symptoms that may be observed in normal pregnancy. ${ }^{5}$ Common physical findings include elevated jugular venous pressure, a displaced apical impulse, and a third heart sound which are similarly non-specific since these are also seen in other forms of heart failure. PPCM is therefore a diagnosis of exclusion and is based on three clinical criteria: manifestation of heart failure toward the end of pregnancy or during the few months following delivery, absence of another identifiable cause of heart failure, and LVEF < 45\%. ${ }^{6}$ In this case, despite the patient's history of hypertension and smoking, her presentation and workup satisfied all three criteria.

Typically, PPCM is managed similarly to that of acute LV systolic heart failure in non-pregnant women with the exception that medications contraindicated during pregnancy (such as angiotensin converting enzyme inhibitors, angiotensin || receptor blockers, and aldosterone antagonists) are avoided. First-line medical therapy includes digoxin, loop diuretics, hydralazine, nitrates, and beta-blockers. ${ }^{5}$ Although pregnancy is a hypercoagulable state, it is not recommended that all peripartum patients receive anticoagulation. However, those with risk factors for venous thromboembolism, such as a low ejection fraction (specifically LVEF < 35\%) - which is associated with an increased risk of LV thrombus - and recent pregnancy should receive anticoagulation with a prophylactic dose of heparin.? Unless patients are acutely decompensating, full-term vaginal deliveries are preferred because of the lower rates of complications such as endometritis and pulmonary embolism. Regional anesthesia (epidural or spinal) can reduce pain and is recommended over general anesthesia, which is associated with myocardial depression. Mothers are encouraged to breastfeed as long as they are hemodynamically stable and there are no contraindications with their medications. ${ }^{8}$
With regards to follow-up, 20-60\% of PPCM patients have complete recovery of LVEF by six months to five years. However, cardiac transplantation, left ventricular assist devices, and implantable cardioverter defibrillators can be considered for women who experience progressive left ventricular dysfunction despite adequate medical therapy and recovery time. ${ }^{7.8}$ Patients who have persistent LVEF $<50 \%$ or LVEF $\leq 25 \%$ at the time of diagnosis should avoid future pregnancies due to the increased risk of heart failure complications, thromboembolic events, and death. ${ }^{6}$ For these patients, a discussion about contraception is imperative. In general, all postpartum patients should avoid estrogencontaining products due to its thrombogenic potential and interference with breastfeeding. Abstinence or sterilization should be heavily counseled for in PPCM patients, but long-acting reversible contraception (LARC) such as progestin-containing intrauterine devices (IUDs)/implants and the copper IUD are also an option. Patients or their partners may also undergo sterilization procedures such as bilateral tubal ligation or vasectomy. ${ }^{6}$ Ultimately, the development of a treatment plan involves the consultation of internists, high-risk obstetricians (maternal fetal medicine), and anesthesiologists.

\section{KEY POINTS}

- This case illustrates the potential for PPCM and acute decompensation in an otherwise healthy patient and the value of recognizing the signs and symptoms associated with this condition, which can be fatal.

- PPCM is a diagnosis of exclusion based on three clinical criteria, and treatment is similar to that of acute systolic LV heart failure.

- Long-term prognosis of PPCM ranges anywhere from full recovery to requiring heart transplantation.

- An understanding of the etiology of PPCM and identification of preventative measures - which have not been thoroughly explored - is clinically significant for obstetricians, cardiologists, anesthesiologists, and neonatologists who may all be involved in either maternal or fetal care. 


\section{REFERENCES}

1. Gunderson, E.P., Croen, L. A., Chiang, V., et al. (2011). Epidemiology of peripartum cardiomyopathy: incidence, predictors, and outcomes. Obstetrics \& Gynecology, 118(3), 583-591.

2. Entresto [prescribing information]. East Hanover, NJ: Novartis Pharmaceuticals Corporation; 2015

3. Hilfiker-Kleiner, D., Kaminski, K., Podewski, E., et al. (2007). A cathepsin D-cleaved $16 \mathrm{kDa}$ form of prolactin mediates postpartum cardiomyopathy Cell, 128(3), 589-600

4. Bültmann, B. D., Klingel, K., Näbauer, M., et al. (2005). High prevalence of viral genomes and inflammation in peripartum cardiomyopathy. American journal of obstetrics and gynecology, 193(2), 363-365.

5. Pearson, G. D., Veille, J. C., Rahimtoola, S., et al. (2000). Peripartum cardiomyopathy: national heart, lung, and blood institute and office of rare diseases (national institutes of health) workshop recommendations and review. Jama, 283(9), 1183-1188.

6. Sliwa, K., HilfikeräKleiner, D., Petrie, M.C., et al. (2010). Current state of knowledge on aetiology, diagnosis, management, and therapy of peripartum cardiomyopathy: a position statement from the Heart Failure Association of the European Society of Cardiology Working Group on peripartum cardiomyopathy. European journal of heart failure, 12(8), 767-778.

7. Blauwet, L. A., \& Cooper, L. T. (2011). Diagnosis and management of peripartum cardiomyopathy. Heart, 97(23), 1970-1981.

8. Elkayam, U. (2011). Clinical characteristics of peripartum cardiomyopathy in the United States: diagnosis, prognosis, and management. Journal of the American College of Cardiology, 58(7), 659-670.

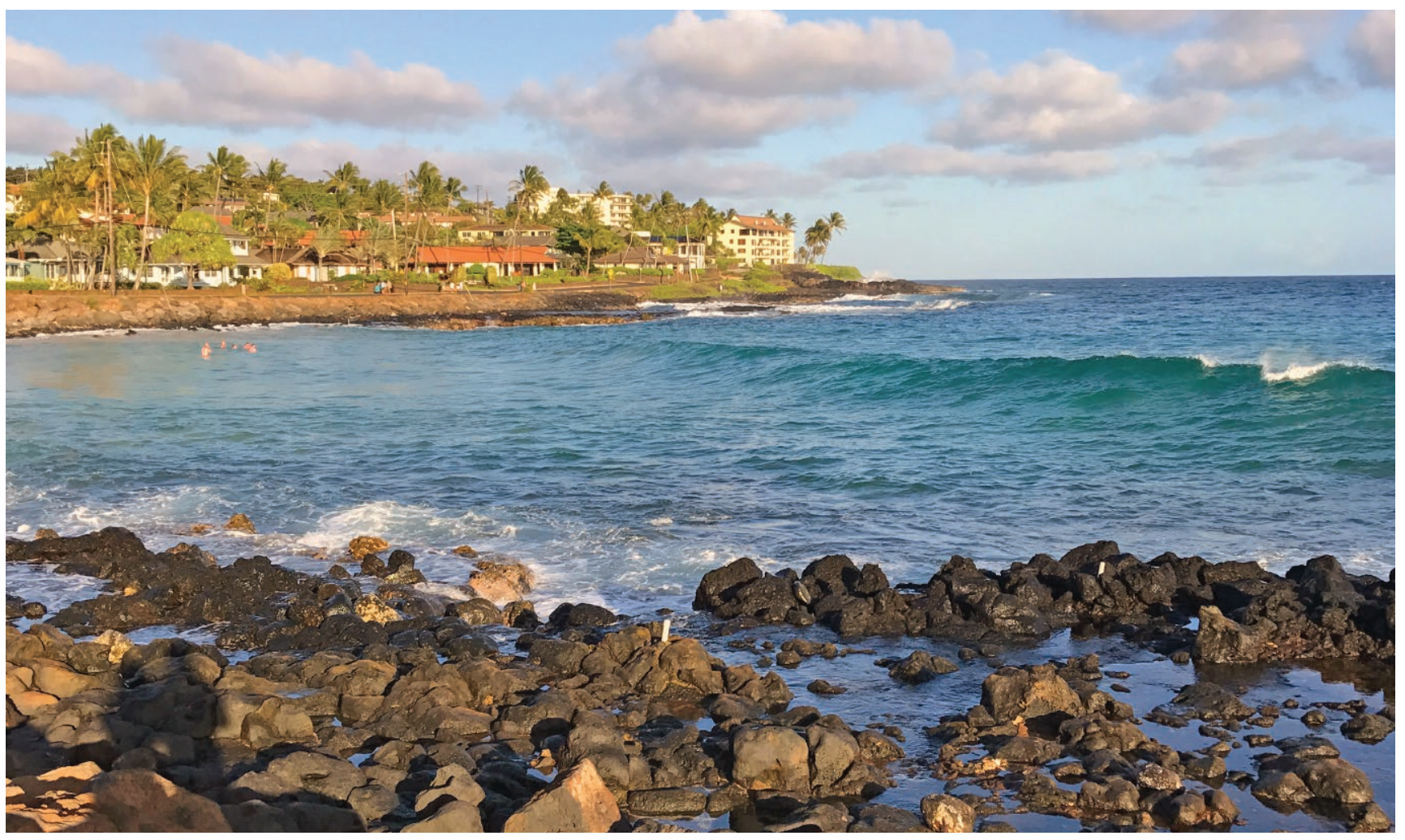

